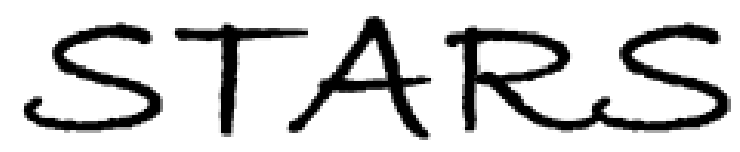

University of Central Florida

STARS

$1-1-2014$

\title{
Numerical evaluation of the fidelity error threshold for the surface code
}

Pejman Jouzdani

University of Central Florida

Eduardo R. Mucciolo

University of Central Florida

Find similar works at: https://stars.library.ucf.edu/facultybib2010 University of Central Florida Libraries http://library.ucf.edu

This Article is brought to you for free and open access by the Faculty Bibliography at STARS. It has been accepted for inclusion in Faculty Bibliography 2010 s by an authorized administrator of STARS. For more information, please contact STARS@ucf.edu.

\section{Recommended Citation}

Jouzdani, Pejman and Mucciolo, Eduardo R., "Numerical evaluation of the fidelity error threshold for the surface code" (2014). Faculty Bibliography 2010s. 5533.

https://stars.library.ucf.edu/facultybib2010/5533

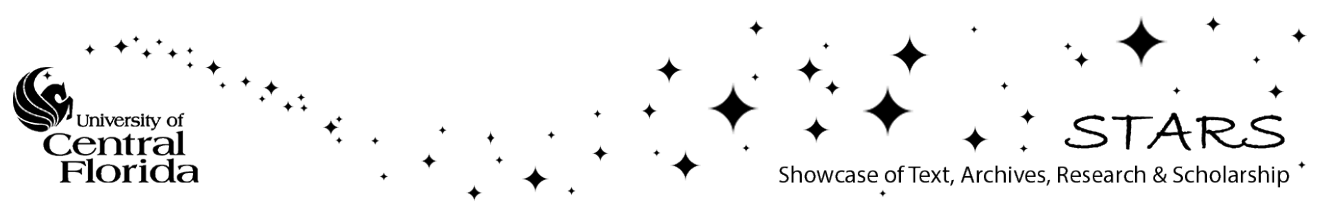




\title{
Numerical evaluation of the fidelity error threshold for the surface code
}

\author{
Pejman Jouzdani and Eduardo R. Mucciolo \\ Department of Physics, University of Central Florida, P. O. Box 162385, Orlando, Florida 32816, USA
}

(Received 4 April 2014; published 11 July 2014)

\begin{abstract}
We study how the resilience of the surface code is affected by the coupling to a non-Markovian environment at zero temperature. The qubits in the surface code experience an effective dynamics due to the coupling to the environment that induces correlations among them. The range of the effective induced qubit-qubit interaction depends on parameters related to the environment and the duration of the quantum error correction cycle. We show numerically that different interaction ranges set different intrinsic bounds on the fidelity of the code. These bounds are unrelated to the error thresholds based on stochastic error models. We introduce a definition of stabilizers based on logical operators that allows us to efficiently implement a Metropolis algorithm to determine upper bounds to the fidelity error threshold.
\end{abstract}

DOI: 10.1103/PhysRevA.90.012315

PACS number(s): 03.67.Lx, 03.67.Pp, 03.65.Yz, 05.50.+q

\section{INTRODUCTION}

Topological quantum codes provide an alternative route to fault-tolerance quantum computation. In topological quantum codes the information is encoded on the topological characteristics of the physical system, resulting in protection against local perturbations [1-4]. The surface (or planar) code [5,6] is an important example of this class of quantum codes that requires an active approach to error correction on lattice of regular qubits. In contrast to the toric code [1], which has an intrinsic Hamiltonian that governs the evolution of the system, the surface code has no intrinsic Hamiltonian and the system's evolution is due to its coupling to an environment and the syndrome extraction and recovery operations at the end of a cycle. It has attracted increasing attention in recent years due to its more practical nature than other topological forms of encodings. Architectures based on superconducting qubits [7] and Majorana fermions [8] have been proposed theoretically. At the experimental level, significant increase in coherence time and fidelity of logical gates in superconducting qubits has been recently reported [9-11], suggesting that these systems may provide a suitable experimental setting for implementing surface codes. Several studies have been done to determine the error threshold of two-dimensional topological codes [12-17]. However, in these studies the role played by correlated errors was not fully investigated. However, it is crucial to study the impact of correlated errors on any scalable quantum code before attempting to quantify error thresholds based on quantum error correction (QEC) protocols [18].

When in contact with environmental degrees of freedom, the physical qubits in the surface code will experience an effective dynamics. This effective dynamics may comprise qubit-qubit interactions, which in turn can result in a correlated time evolution. Since a large-scale quantum code has a large Hilbert space, a correlated dynamics may cause a sharp change in the quantum phase of the code system, even in the presence of QEC operations. This change of phase cannot be studied in the context of stochastic noise models, which typically only include bit flip, phase flip, and depolarizing channels.

The effective dynamics induced by the environment on the code system is in general very nontrivial to derive from first principles. However, for a particular case, the bosonic bath, we were able to obtain an exact effective action after a single QEC cycle $[19,20]$. This action comprises a qubit-qubit interaction term with a distance-dependent exchange coupling. The range and strength of qubit-qubit interaction were found to be functions of environmental parameters, the distance between the qubits, and the duration of the QEC cycle. The effective dynamics derived for a bosonic bath could be used as a phenomenological model for other types of environments as it has a rather general functional form.

In this paper we numerically evaluate the effect of correlated errors induced by a two-qubit effective action and study the impact of different ranges of correlations. We use a Monte Carlo method for evaluating the fidelity of the surface code at the end of a complete QEC cycle. We introduce an alternative approach to define the surface code stabilizers that helps us to implement an efficient Metropolis algorithm. This method can be extended to other topological systems such as the toric code. For the surface code, we confirm the results presented in Ref. [25], namely, the existence of a sharp transition in the fidelity as a function of the coupling between qubits and the environment for large enough codes. The critical value of this coupling provides a threshold for the ability of the surface code to protect quantum information. We also find that an increase in the correlation range does not wash away this critical point but moves it to lower coupling constant values, making it more difficult in practice to achieve protection.

The paper is organized as follows. In Sec. II we introduced the basic elements of the surface code. In Sec. III we describe a model interaction for the environment and the physical qubits that induces an effective evolution for the code system. We then use this evolution in Sec. IV to obtain an expression for the surface code fidelity in terms of expectation values of a spin statistical model. The numerical Monte Carlo method used to compute these expectation values and the results are described in Secs. V and VI. Finally, a summary is provided in Sec. VII.

\section{SURFACE CODE}

The surface code [5,6] is a collection of $N$ qubits located on the links of a two-dimensional lattice, as shown in Fig. 1. There are two types of stabilizers, $\hat{A}_{s}$ and $\hat{B}_{p}$, which are defined as

$$
\hat{A}_{s}=\prod_{i \in s} \sigma_{i}^{x},
$$




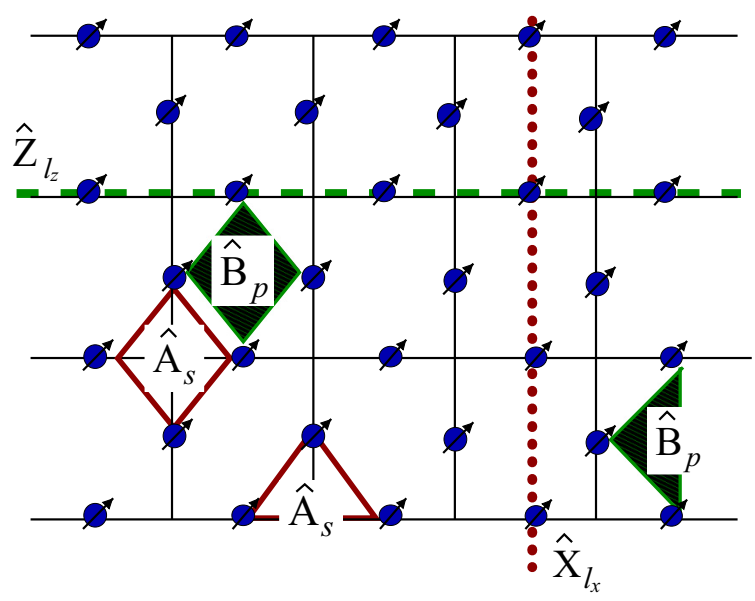

FIG. 1. (Color online) The geometry of the surface code system. Physical qubits are shown with arrows. A plaquette (star) operator $\hat{B}_{p}\left(\hat{A}_{s}\right)$ is shown with a shaded (unshaded) enclosed area connecting the corresponding qubits. The dashed green (dotted red) line $l_{z}\left(l_{x}\right)$ represents the logical operator $\hat{Z}_{l_{z}}\left(\hat{X}_{l_{x}}\right)$ and runs over corresponding qubits.

and

$$
\hat{B}_{p}=\prod_{i \in p} \sigma_{i}^{z} .
$$

They have eigenvalues $A_{s}$ and $B_{p}$, respectively, that take the values \pm 1 . The subscript $s(p)$ refers to a vertex (plaquette) on the lattice, and $\sigma_{i}^{\alpha}$ is the $\alpha$ component of the Pauli matrices that acts on the $i$ th qubit. The two stabilizers commute, $\left[\hat{A}_{s}, \hat{B}_{p}\right]=0$, and thus are simultaneously observable. In addition, there are two logical operators defined as

$$
\hat{X}_{l_{x}}=\prod_{i \in l_{x}} \sigma_{i}^{x},
$$

and

$$
\hat{Z}_{l_{z}}=\prod_{i \in l_{z}} \sigma_{i}^{z},
$$

where the path $l_{x}\left(l_{z}\right)$ runs from one boundary to the opposite boundary, as shown in Fig. 1. The two logical operators follow the same commutation relations as the Pauli matrices $\sigma^{x}$ and $\sigma^{z}$ and both commute with the stabilizers in Eqs. (1) and (2).

The code space is defined as the particular subspace of the total Hilbert space of the system for which the outcome of any stabilizer is +1 . The maximum set of observables can be either $\{\{\hat{A}\},\{\hat{B}\}, \hat{Z}\}$ or $\{\{\hat{A}\},\{\hat{B}\}, \hat{X}\}$. Considering the set $\{\{\hat{A}\},\{\hat{B}\}, \hat{Z}\}, \hat{X}$ anticommutes with the logical operator in the set, $\hat{Z}$. Therefore, there are only two distinct basis states $|S C\rangle$ and $\hat{X}|S C\rangle$ that satisfy the condition of the code space. $\hat{X}$ can be chosen along different paths $l_{x}$. However $\hat{X}|S C\rangle$ is unique and independent of the chosen logical operator. These two orthogonal states are the two states of the logical qubit of the surface code. The code is topologically protected, e.g., to flip the logical qubit state $|S C\rangle$ to $\hat{X}|S C\rangle$ a logical operator comprising at least $L$ physical qubit operations must be applied, where $L$ is the linear size of the system. We will refer to $|S C\rangle$ as the code state.
Any deviation from the code space due to local errors such as qubit flips or phase flips results in excitations known as anyons. The anyons correspond to stabilizers that yield an outcome -1 after measurement. Anyons corresponding to $\hat{A}$ stabilizers are referred to as " $e$ " type, while anyons corresponding to $\hat{B}$ stabilizers are " $m$ " type.

\section{INTERACTION WITH THE ENVIRONMENT}

When the system is in contact with a bath the total Hamiltonian is

$$
H=H_{B}+V_{B C}
$$

where $H_{B}$ is the bath Hamiltonian and $V_{B C}$ is the interaction part. If the closed system is prepared in the product state $|S C\rangle \otimes|B\rangle$, where the $|B\rangle$ is the bath ground state, the closed system evolves in time as

$$
|\psi(t)\rangle=U_{I}(t)|S C\rangle \otimes|B\rangle,
$$

where $U_{I}(t)$ is the time evolution operator of the combined system in the interaction picture. At the end of the QEC cycle the state of the environment may have components beyond its ground state. As a result, the entanglement between the qubits and the environment can spill over to the next QEC cycle. While this effect deserves investigation, here we will adopt a simplifying hypothesis and assume that the environment remains in its ground state at the end of the QEC cycle. This could be achieved by maintaining the environment cold (i.e., by keeping it in contact with a much larger bath or reservoir). Hence, we define

$$
U_{\text {eff }}(\Delta)=\left\langle B\left|U_{I}(\Delta)\right| B\right\rangle,
$$

as the effective evolution operator of the code system at the end of a QEC cycle of duration $\Delta$.

The evolution under $U_{\text {eff }}(\Delta)$ induces an effective dynamics into the code system that includes dissipation and dephasing. In general, the functional form of $U_{\text {eff }}(\Delta)$ in terms of the qubit operators $\left\{\sigma_{i}^{\alpha}\right\}$ can be rather difficult to derive from first principles. For the particular case of a gapless bosonic environment with a coupling given by

$$
V_{B C}=\lambda \sum_{r_{i}} f\left(r_{i}\right) \sigma_{i}^{x},
$$

a simple expression can be exactly derived. Here, $\lambda$ is the strength of coupling to the bosonic field, $f\left(r_{i}\right)$ is the bosonic field operator of the bath, and $\sigma_{i}^{x}$ is the Pauli matrix acting on the $i$ th qubit. In this case, the induced evolution operator dynamics was found to be $[19,20]$

$$
U_{\mathrm{eff}}(\Delta)=e^{\beta H_{\mathrm{eff}}}=e^{-\beta \sum_{i j} J_{i j} \sigma_{i}^{x} \sigma_{j}^{x}} .
$$

The sum in the exponent is over the physical qubits of the surface code (see Fig. 2). The parameter $\beta$ is a function of $\lambda$ and other characteristics of the bosonic environment. For the Ohmic case [20],

$$
\beta=\frac{1}{2 \pi}\left(\frac{\lambda}{\omega_{0}}\right)^{2}
$$




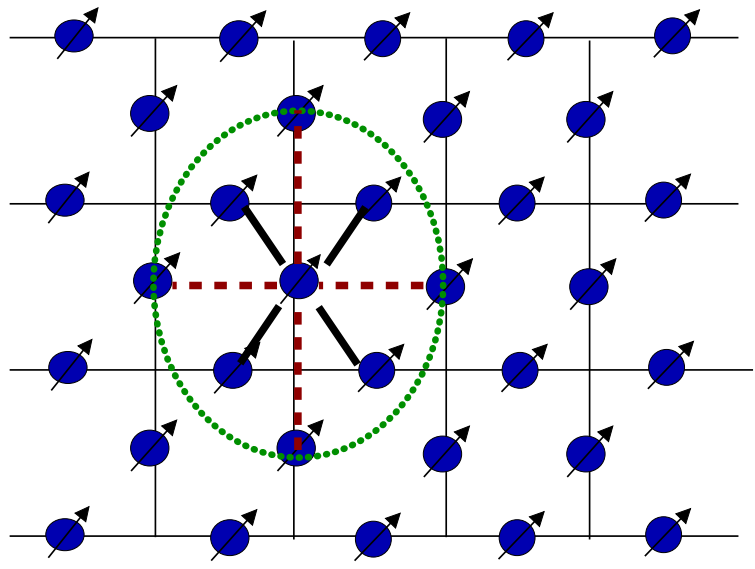

FIG. 2. (Color online) The effective interaction induced by the bath, Eq. (9), between a qubit and its nearest (solid black lines) and next-to-nearest neighbors (dashed red lines). The range of the interaction (dotted circle) is related to the QEC period $\Delta$.

with $\omega_{0}$ denoting a characteristic frequency of the bosons and

$$
J_{i j}=\frac{1}{2} \times \begin{cases}\operatorname{arcosh}\left(\frac{v \Delta}{\left|\mathbf{r}_{i}-\mathbf{r}_{j}\right|}\right)+\frac{i \pi}{2}, & \frac{\left|\mathbf{r}_{i}-\mathbf{r}_{j}\right|}{v \Delta}<1, \\ i \arcsin \left(\frac{v \Delta}{\left|\mathbf{r}_{i}-\mathbf{r}_{j}\right|}\right), & \frac{\left|\mathbf{r}_{i}-\mathbf{r}_{j}\right|}{v \Delta}>1 .\end{cases}
$$

Here $v$ is the bosonic mode velocity. The complex interaction $J_{i j}$ is directly related to the correlation function of the bath at two spacial points $\mathbf{r}_{i}$ and $\mathbf{r}_{j}$ and to $\Delta$. Notice that Eq. (11) was derived in Ref. [20] under the assumption that the bath returns to its ground state at the end of the QEC cycle, as shown in Eq. (7).

The functional form in the effective action in Eq. (9) can also be used as a phenomenological error model of correlated errors with a complex exchange coupling parameter $J_{i j}$. We note that other forms of interaction between the surface code constituents and the environment have been used in the literature. In particular, one may start with an interaction such as $V_{B C} \sim \lambda \sum_{r_{i}} f\left(r_{i}\right) A_{i}$ where a bosonic field couples to the stabilizers. In this case, the resulting effective dynamics may enhance the surface code protection [21,22].

\section{QEC WITH FLAWLESS RECOVERY}

The system is maintained in its code space by means of QEC cycles. At each QEC cycle the stabilizers are measured (syndrome detection) and a suitable recovery operation is performed with the goal of returning the system to its code state, as defined in Sec. II. Due to the interaction with the environment, prior to the syndrome detection the code system is in a superposition state where all syndromes are possible. After reading the stabilizers, the system is detected (i.e., projected) onto a particular superposition state (syndrome). Eventually, a nondestructive recovery returns the system back to the code state, $|S C\rangle$, or erroneously to $\hat{X}|S C\rangle$.

The syndromes in the QEC protocol are based on measurements of the stabilizers. Let us define $\mathcal{P}_{n, f}=|n, f\rangle\langle n, f|$ as the projection onto a subspace of $n$ excitations or anyons. The $n$ excitations may be detected at different vertices or plaquettes on the surface code. The index $f$ refers to the configuration where anyons are detected on the surface code after syndrome detection. Due to the topological nature of the code, any state $|n, f\rangle$ is a superposition of a large number of states involving the physical qubits of the system,

$$
|n, f\rangle=\sum_{s^{*}}\left|s^{*}\right\rangle
$$

where the sum is over configurations of the physical qubits $\left|s^{*}\right\rangle=\left|s_{1}, \ldots, s_{N}\right\rangle$ and the asterisk indicates that the sum is taken over the configurations that are consistent with the condition of " $n$ anyons with the configuration $f$."

After being initially set in the code state $|S C\rangle$, the system interacts with the environment. After a time interval $\Delta$, it evolves to the state $U_{\text {eff }}(\Delta)|S C\rangle$. The effective time evolution operator $U_{\text {eff }}(\Delta)$ is in general nontrivial and may not be unitary. At this point we assume that the QEC operation detects the system (with some probability) to be in the state $|n, f\rangle$. Then, a flawless recovery operation $\mathcal{R}$ returns this state to either $|S C\rangle$ or, erroneously, to $\hat{X}|S C\rangle$, namely,

$$
\mathcal{R} \mathcal{P}_{n, f} U_{\text {eff }}(\Delta)|S C\rangle=\mathcal{A}|S C\rangle+\mathcal{B} \hat{X}|S C\rangle,
$$

where $\mathcal{A}$ and $\mathcal{B}$ are the amplitudes of the two orthogonal states $|S C\rangle$ and $\hat{X}|S C\rangle$, respectively.

To be more explicit, let us exactly specify the projector $\mathcal{P}_{n, f}=|n, f\rangle\langle n, f|$ for the case where errors are of " $m$ " type; " $m$ " type errors occur as a result of bit flipping qubits along a set of strings. We define this string operation as

$$
\hat{S}^{x}(\mathcal{L})=\prod_{j \in \mathcal{L}} \sigma_{j}^{x}
$$

where $\mathcal{L}$ is a set of strings running on the surface code lattice such that $|n, f\rangle=\hat{S}^{x}(\mathcal{L})|S C\rangle$. There are many possible sets of $\mathcal{L}$ and corresponding $\hat{S}^{x}(\mathcal{L})$ that generate the same state $|n, f\rangle$. Two such choices of string operators, $\hat{S}^{x}\left(\mathcal{L}_{1}\right)$ and $\hat{S}^{x}\left(\mathcal{L}_{2}\right)$, can differ from each other in two possible ways: Either

$$
\hat{S}^{x}\left(\mathcal{L}_{1}\right)|S C\rangle=\left[\prod_{s \in \mathcal{P}} \hat{A}_{s}\right] \hat{S}^{x}\left(\mathcal{L}_{2}\right)|S C\rangle=\hat{S}^{x}\left(\mathcal{L}_{2}\right)|S C\rangle,
$$

or

$$
\begin{aligned}
\hat{S}^{x}\left(\mathcal{L}_{1}\right)|S C\rangle & =\left[\prod_{s \in \mathcal{P}} \hat{A}_{s} \hat{X}\right] \hat{S}^{x}\left(\mathcal{L}_{2}\right)|S C\rangle \\
& =\hat{X}^{\prime} \hat{S}^{x}\left(\mathcal{L}_{2}\right)|S C\rangle .
\end{aligned}
$$

Here, $\mathcal{P}$ is a set of vertices on the surface code. In Eqs. (15) and (16) we used the identity $\left[\prod_{s \in \mathcal{P}} \hat{A}_{s}\right]|S C\rangle=|S C\rangle$. Thus, the two states $S^{x}(\mathcal{L})|S C\rangle$ and $\hat{X} S^{x}(\mathcal{L})|S C\rangle$ alone are enough to define $\mathcal{P}_{n, f}$ as

$$
\begin{aligned}
\mathcal{P}_{n, f}= & \hat{S}^{x}(\mathcal{L})|S C\rangle\langle S C| \hat{S}^{x}(\mathcal{L}) \\
& +\hat{X} \hat{S}^{x}(\mathcal{L})|S C\rangle\langle S C| \hat{S}^{x}(\mathcal{L}) \hat{X}
\end{aligned}
$$

With this definition we find the state of the code at the end of the first QEC cycle to be

$$
\begin{aligned}
|S C(\Delta)\rangle= & \mathcal{R} \mathcal{P}_{n, f} U_{\mathrm{eff}}(\Delta)|S C\rangle \\
& +\mathcal{R} \hat{S}^{x}(\mathcal{L})|S C\rangle\left\langle S C\left|\hat{S}^{x}(\mathcal{L}) U_{\mathrm{eff}}(\Delta)\right| S C\right\rangle \\
& +\mathcal{R} \hat{X} \hat{S}^{x}(\mathcal{L})|S C\rangle\left\langle S C\left|\hat{S}^{x}(\mathcal{L}) \hat{X} U_{\mathrm{eff}}(\Delta)\right| S C\right\rangle \\
= & \mathcal{A}_{n, p}|S C\rangle+\mathcal{B}_{n, p} \hat{X}|S C\rangle
\end{aligned}
$$


where we assume a flawless recovery, $\mathcal{R} \hat{S}^{x}(\mathcal{L})=\mathbf{1}$, and define the amplitudes,

$$
\mathcal{A}_{n, f}=\left\langle S C\left|\hat{S}^{x}(\mathcal{L}) U_{\text {eff }}(\Delta)\right| S C\right\rangle,
$$

and

$$
\begin{aligned}
\mathcal{B}_{n, f} & =\left\langle S C\left|\hat{S}^{x}(\mathcal{L}) \hat{X} U_{\text {eff }}(\Delta)\right| S C\right\rangle \\
& =\left\langle S C\left|\hat{S}^{x}(\overline{\mathcal{L}}) U_{\text {eff }}(\Delta)\right| S C\right\rangle .
\end{aligned}
$$

The fidelity is a suitable quantity to measure the success of the QEC operation after error correction,

$$
\mathcal{F}=\frac{\langle S C \mid S C(\Delta)\rangle}{\sqrt{\langle S C(\Delta) \mid S C(\Delta)\rangle}} .
$$

It is straightforward to show that the fidelity can be written in terms of the amplitudes $\mathcal{A}_{n, f}$ and $\mathcal{B}_{n, f}$, namely,

$$
\mathcal{F}=\frac{1}{\sqrt{1+\left|\frac{\mathcal{B}_{n, f}}{\mathcal{A}_{n, f}}\right|^{2}}}
$$

In order to find a suitable expression for the numerical evaluation of the amplitudes $\mathcal{A}_{n, f}$ and $\mathcal{B}_{n, f}$, we write $|S C\rangle$ as

$$
|S C\rangle=\frac{1}{\sqrt{2^{N_{\diamond}}}} \prod_{s}\left(1+\hat{A}_{s}\right)\left|F_{z}\right\rangle,
$$

where $\left|F_{z}\right\rangle$ is the ferromagnet state of the qubits in the $z$ direction: $\left|F_{z}\right\rangle=|\uparrow\rangle_{1} \ldots|\uparrow\rangle_{N}$, with $N_{\diamond}$ being number of star operators. Noting that

$$
\left|F_{z}\right\rangle=\prod_{i=1}^{N} \frac{|+\rangle_{i}+|-\rangle_{i}}{\sqrt{2}},
$$

where $\sigma_{i}^{x}| \pm\rangle_{i}= \pm 1| \pm\rangle_{i}$ and $| \pm\rangle_{i}$ stands for eigenvectors of Pauli matrix $\sigma_{i}^{x}$ acting on $i$ th physical qubit, we obtain

$$
|S C\rangle=\frac{1}{\sqrt{2^{N_{\diamond}-1}}} \sum_{s^{*}}\left|s^{*}\right\rangle .
$$

The sum in Eq. (25) runs over restricted states $s^{*}$ (a product state of $| \pm\rangle_{i}$ of physical qubits) that preserve the conditions $A_{s}=1$ (i.e., $\hat{A}_{s}\left|s^{*}\right\rangle=+\left|s^{*}\right\rangle$ ) for all vertices $s$ of the lattice. The state $|S C\rangle$ also satisfies the conditions $\hat{Z}|S C\rangle=+|S C\rangle$ and $B_{p}=1\left(\hat{B}_{p}|S C\rangle=+|S C\rangle\right)$ for all plaquettes of the lattice. Hereafter we will make use of the relations,

$$
\hat{S}^{x}(\mathcal{L})\left|s^{*}\right\rangle=S_{s^{\star}}(\mathcal{L})\left|s^{*}\right\rangle,
$$

and

$$
\hat{S}^{x}(\mathcal{L}) \hat{X}\left|s^{*}\right\rangle=S_{s^{*}}(\overline{\mathcal{L}})\left|s^{*}\right\rangle,
$$

with $S_{S^{\star}}= \pm 1$ being the product of the $\sigma_{i}^{x}$ operators along either the path $\mathcal{L}$ or $\overline{\mathcal{L}}$. By inserting Eqs. (25)-(27) into Eqs. (19) and (20), we arrive at

$$
\mathcal{B}=\frac{\mathcal{B}_{n, f}}{\mathcal{A}_{0,0}}=\frac{\sum_{s^{*}} S_{s^{\star}}(\overline{\mathcal{L}}) U_{\text {eff }}\left(s^{\star}\right)}{\sum_{s^{*}} U_{\text {eff }}\left(s^{\star}\right)},
$$

and

$$
\mathcal{A}=\frac{\mathcal{A}_{n, f}}{\mathcal{A}_{0,0}}=\frac{\sum_{s^{*}} S_{s^{\star}}(\mathcal{L}) U_{\text {eff }}\left(s^{\star}\right)}{\sum_{s^{*}} U_{\text {eff }}\left(s^{\star}\right)} .
$$

Here $U_{\text {eff }}\left(s^{\star}\right)$ is the matrix element $\left\langle s^{\star}\left|U_{\text {eff }}(\Delta)\right| s^{\star}\right\rangle$ that can be considered as a statistical weight in the sums shown above.
Equations (28) and (29) show that the calculation of $\mathcal{A}_{n, f}$ and $\mathcal{B}_{n, f}$ maps onto a statistical mechanics problem where these amplitudes are equal to the expectation values $\langle S(\mathcal{L})\rangle$ and $\langle S(\overline{\mathcal{L}})\rangle$, respectively. The averaging $\langle\ldots\rangle$ is performed with respect to a complex-time effective action $H_{\text {eff }}$ that gives rise to the statistical weight $U_{\text {eff }}\left(s^{*}\right)$ introduced above. In the following, we study the fidelity of the code based on the behavior of the amplitudes $\mathcal{B}$ and $\mathcal{A}$ for an effective action comprising qubit-qubit interactions of the form introduced in Eq. (9). We limit our study to real values of $\beta$ and $J_{i j}$ while probing different ranges of interactions, namely nearest neighbors and next-to-nearest neighbors. We remark that the range of the effective qubit-qubit interaction can be sharply controlled by the duration of the QEC cycle: Longer cycles lead to longer ranges while shorter cycles decrease the range, even down to nearest neighbors.

\section{NUMERICAL METHOD}

We numerically evaluate the amplitudes $\mathcal{A}$ and $\mathcal{B}$, as defined in Eqs. (28) and (29), using a classical Monte Carlo method and assuming an effective evolution operator as in Eq. (9), with an effective action of the form,

$$
H_{\mathrm{eff}}=-\sum_{(i j)} J\left(\left|r_{i}-r_{j}\right|\right) \sigma_{i}^{x} \sigma_{j}^{x},
$$

for nearest-neighbor and beyond nearest-neighbor interactions.

Using the standard classical Monte Carlo method [23], we replace the summation over the large set of configurations $\left\{s^{*}\right\}$ in Eqs. (28) and (29), which is of order $\left[O\left(2^{\frac{N}{2}}\right)\right]$, by a sum over a smaller set of representative sample configurations $\{\tau\}$ for a given value of $\beta J$. All the sampled configurations have the same statistical weight $e^{-\beta E_{\tau}}$. If there are $M$ representative configurations for a given $\beta J$, we then have

$$
\sum_{s^{*}}\{\ldots\} e^{-\beta E_{s^{*}}} \rightarrow \frac{1}{M} \sum_{\tau}\{\ldots\},
$$

where we target the average value of the quantity $\{\ldots\}$. We use a Metropolis algorithm to collect these relevant configurations assuming that $\beta J$ is real. However, since we must take into account the constraint $A_{s}=1$ for any vertex $s$, the standard Metropolis algorithm needs to be suitably modified.

In general, a state $\left|s^{*}\right\rangle$ that satisfies the constraint " $A_{s}=1$ for all $s$ " in Eq. (25) has the form,

$$
\left|s^{*}\right\rangle=\left\{\begin{array}{l}
\prod_{p \in \mathcal{P}} \hat{B}_{p}\left|F_{x}\right\rangle, \\
\text { or } \\
\prod_{p \in \mathcal{P}} \hat{B}_{p} \hat{Z}\left|F_{x}\right\rangle .
\end{array}\right.
$$

Here, $\mathcal{P}$ is an arbitrary set of plaquettes. States in the first class, (I), are eigenstates of $\hat{X}$ with eigenvalue +1 , while in the second class, (II), the states are eigenstates of $\hat{X}$ with eigenvalue -1 . Equation (32) provides a natural codification of the restricted states $\left|s^{*}\right\rangle$ : One can start with a vacuum state $\left|F_{x}\right\rangle$, then flip a number of qubits by $\prod_{p \in \mathcal{P}} \hat{B}_{p}$ that correspond to $\mathcal{P}$, and arrive at a restricted state $\left|s^{*}\right\rangle$. However, this is not the route we pursue. Below we present an equivalent but alternative definition for the stabilizers of the surface code 


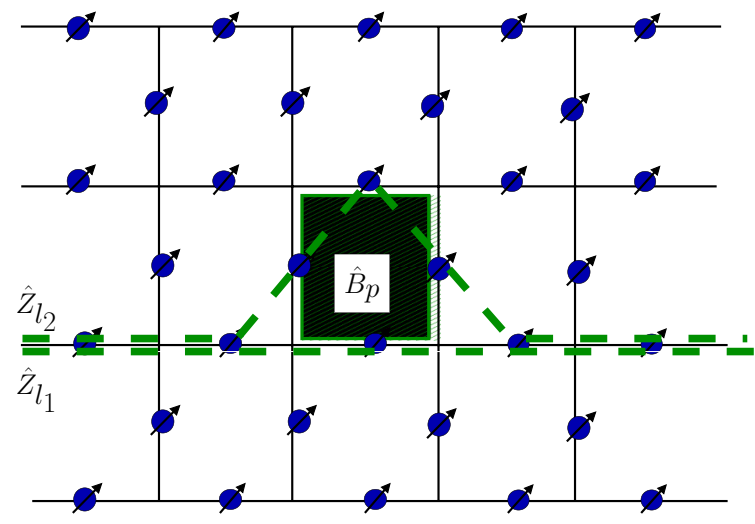

FIG. 3. (Color online) Applying two logical $\hat{Z}$ operators along the paths $l_{1}$ and $l_{2}$ is equivalent to a $\hat{B}_{p}$ operator shown with the hatched rectangle.

and consequently of the states in Eq. (32). They provide a more efficient implementation of the Metropolis algorithm. The alternative definition for stabilizers is not limited to the surface code and can be extended to higher-dimensional codes.

Since $\sigma_{i}^{\alpha} \sigma_{i}^{\alpha}=1$ for $\alpha=x, y, z$, one can write the stabilizers of Eqs. (1) and (2) as

$$
\hat{A}_{s}=\prod_{i \in s} \sigma_{i}^{x}=\hat{X}_{l_{1}} \hat{X}_{l_{2}} \ldots \hat{X}_{l_{2 m}}
$$

and

$$
\hat{B}_{p}=\prod_{i \in p} \sigma_{i}^{z}=\hat{Z}_{l_{1}} \hat{Z}_{l_{2}} \ldots \hat{Z}_{l_{2 m}},
$$

where a path $l_{i}$ goes from one boundary to the opposite boundary of the system. The set $\left\{l_{i}\right\}$ is chosen such that the product of the logical operators $\hat{X}_{l_{1}} \hat{X}_{l_{2}} \ldots \hat{X}_{l_{2 m}}$ (or $\hat{Z}_{l_{1}} \hat{Z}_{l_{2}} \ldots \hat{Z}_{l_{2 m}}$ ) is equal to $\prod_{i \in s} \sigma_{i}^{x}$ (or $\prod_{i \in p} \sigma_{i}^{z}$ ) and thus the stabilizer $\hat{A}_{s}$ (or $\hat{B}_{p}$ ). The number of paths, $2 m$, is not unique. An even number guarantees that the commutation relation $\left[\hat{A}_{s}, \hat{B}_{p}\right]=0$ is satisfied. The product of $2 m$ logical operators always forms closed loops. For example, in Fig. 3 the operation of the stabilizer operator $\hat{B}_{p}$ on the qubits of plaquette $p$ is generated by applying two logical $\hat{Z}$ operators along the paths $l_{1}$ and $l_{2}$, as depicted in the figure.

Thus, the states $\left|s^{*}\right\rangle$ defined in Eq. (32) can be stated in terms of logical operations as

$$
\left|s^{*}\right\rangle=\left\{\begin{array}{l}
\hat{Z}_{l_{1}} \hat{Z}_{l_{2}} \ldots \hat{Z}_{l_{2 m}}\left|F_{x}\right\rangle=\mathbf{Z}_{l}^{2}\left|F_{x}\right\rangle, \\
\text { or } \\
\hat{Z}_{l_{1}} \hat{Z}_{l_{2}} \ldots \hat{Z}_{l_{2 m}} \hat{Z}\left|F_{x}\right\rangle=\mathbf{Z}_{l}\left|F_{x}\right\rangle,
\end{array}\right.
$$

where we abbreviate the product $\hat{Z}_{l_{1}} \hat{Z}_{l_{2}} \ldots \hat{Z}_{l_{2 m}}$ as $\mathbf{Z}_{l}^{2}$ and $\hat{Z}_{l_{1}} \hat{Z}_{l_{2}} \ldots \hat{Z}_{l_{2 m}} \hat{Z}$ as $\mathbf{Z}_{l}$. By introducing these definitions into Eqs. (28) and (29) and using the fact that $\mathbf{Z}_{l}^{2} \hat{X}=\hat{X} \mathbf{Z}_{l}^{2}$ and $\mathbf{Z}_{l} \hat{X}=-\hat{X} \mathbf{Z}_{l}$, we arrive at

$$
\begin{aligned}
\mathcal{B}= & \sum_{l}\left\{\left\langle F_{x}\left|\mathbf{Z}_{l}^{2} S^{x}(\mathcal{L}) U_{\text {eff }} \mathbf{Z}_{l}^{2}\right| F_{x}\right\rangle\right. \\
& \left.-\left\langle F_{x}\left|\mathbf{Z}_{l} S^{x}(\mathcal{L}) U_{\text {eff }} \mathbf{Z}_{l}\right| F_{x}\right\rangle\right\},
\end{aligned}
$$

and

$$
\begin{aligned}
\mathcal{A}= & \sum_{l}\left\{\left\langle F_{x}\left|\mathbf{Z}_{l}^{2} S^{x}(\mathcal{L}) U_{\text {eff }} \mathbf{Z}_{l}^{2}\right| F_{x}\right\rangle\right. \\
& \left.+\left\langle F_{x}\left|\mathbf{Z}_{l} S^{x}(\mathcal{L}) U_{\text {eff }} \mathbf{Z}_{l}\right| F_{x}\right\rangle\right\},
\end{aligned}
$$

up to a common normalization factor.

To understand the essential difference between $\mathcal{A}$ and $\mathcal{B}$, let us assume a phase of the system in which the states belonging to the two classes of Eq. (35) contribute with the same statistical weight $U_{\text {eff }}$ (the topological state). In this phase any fluctuation around the equilibrium configuration states, $\left\{\left|s^{\star}\right\rangle\right\}$ (which is of the order of $\mathcal{L}$ and less than the distance of the code $\frac{L}{2}$ ), will be canceled out in the sum in the expression for $\mathcal{B}$ via the minus sign of the second term in Eq. (36). Hence, the ratio $\left|\frac{\mathcal{B}}{\mathcal{A}}\right| \rightarrow 0$ in the thermodynamic limit and the fidelity $\mathcal{F} \rightarrow 1$, as expected. However, in the phase where the statistical weight of the states in class I differs from states in class II in Eq. (35), i.e., in the ordered phase, there is a sizable change in $\left|\frac{\mathcal{B}}{\mathcal{A}}\right|$. In our model the ordered phase corresponds to the state $\left|F_{x}\right\rangle$. In this limit, $\left|\frac{\mathcal{B}}{\mathcal{A}}\right| \rightarrow 1$ and a sharp phase transition takes place between these two limits. Thus, for a correct decoding and sufficiently large system, one should expect to see $|\mathcal{B}|<|\mathcal{A}|$ in the disordered phase (topological phase) in a universal way, independently of the error $S_{S^{\star}}(\mathcal{L})$, as long as $\mathcal{L}<\frac{L}{2}$.

Equation (35) provides a novel way for the codification of the restricted state $\left|s^{*}\right\rangle$ : One begins with a vacuum state $\left|F_{x}\right\rangle$, then flips a number of qubits along a certain path $\left\{l \equiv l_{1} \ldots\right\}$, and arrives at a restricted state $\mathbf{Z}_{l}^{2}\left|F_{x}\right\rangle$ or $\mathbf{Z}_{l}\left|F_{x}\right\rangle$. The sums in Eqs. (36) and (37) run over all possible paths $\{l\}$. The statistical weight $U_{\text {eff }}$ corresponds to the probability of flipping the qubits along the path $l$. In this regard, the Metropolis algorithm finds the most relevant paths. The scheme to update the configurations is then similar to the techniques used in world-line-based quantum Monte Carlo [24], since each two $\mathbf{Z}_{l}$ and $\mathbf{Z}_{l^{\prime}}$ differ by a certain number of $\hat{B}_{p}$ operations.

\section{NUMERICAL RESULTS}

Considering a real homogeneous interaction coupling $J_{i j}=J\left(\left|r_{i}-r_{j}\right|\right)$, we use the method described in Sec. V to numerically evaluate $\mathcal{A}, \mathcal{B}$, and $\left|\frac{\mathcal{B}}{\mathcal{A}}\right|$ to determine the fidelity $\mathcal{F}$.

\section{A. Results for the nonerror sector $\mathcal{P}_{\mathbf{0 , 0}}$}

For the no-charge sector $\mathcal{P}_{0,0}$ we have $S^{x}(\mathcal{L})=\mathbf{1}$. Our numerical results show that $\left|\frac{\mathcal{B}}{\mathcal{A}}\right|$ follows the local order parameter $\left\langle\sigma_{i}^{x}\right\rangle$, where $i$ is an arbitrary qubit in the bulk of the surface code system. The behavior of $\left|\frac{\mathcal{B}}{\mathcal{A}}\right|$ as a function of $\beta J$ for nearest-neighbor interaction and different system sizes is shown in Fig. 4.

The surface code geometry can be decomposed into two sublattices. Here we considered square sublattices of sizes $L \times L$ and $(L-1) \times(L-1)$. By increasing the system size the transition from the topological state, where $\beta J<\beta_{c} J$, to a trivial state where the degeneracy between $\left|s^{*}\right\rangle$ states is lifted, becomes sharper. This confirms the first-order phase transition nature of the effect (i.e., the existence of an error threshold 


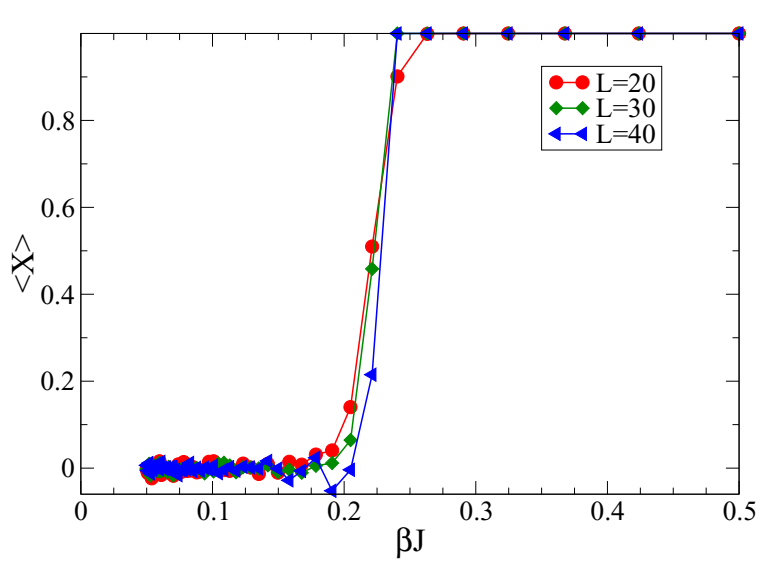

FIG. 4. (Color online) The numerical evaluation of $\langle X\rangle=\left|\frac{\mathcal{B}}{A}\right|$ for nonerror syndromes based on the Monte Carlo calculation for different system sizes. $L=20$ is a surface code system with 761 qubits (circle), $L=30$ has 1741 qubits (diamond), and $L=40$ has 3121 qubits. The solid lines are guides to the eye. On the horizontal axis, $\beta$ is proportional to the coupling to the environment and $J$ is the exchange coupling of the effective interaction between nearest-neighbor qubits. In this simulation 80000 iterations are used for each $\beta$ step.

in the fidelity). A finite-size scaling of the heat capacity is shown in Fig. 5. Taking $\beta$ as a fictitious inverse temperature, we used $\frac{\beta^{2}\left(\left\langle E^{2}\right\rangle-\langle E\rangle^{2}\right)}{V}$ as the definition of heat capacity. Here $V$ is the total number of qubits. By setting $J=1$ and fitting the data to the asymptotic functional form $\beta_{c}(L)=\beta_{c}(\infty)-y L^{x}$, we find the critical exponent $x=-1 / v=-1$, in agreement with the expected Ising model $(v=1)$. Scaling the data according to this functional form also gives $\beta_{c}(\infty)=0.217$. This value agrees closely with the analytical result obtained in Ref. [25].

In this one-cycle study, the time period of the cycle, $\Delta$, affects directly the range of interaction in $H_{\text {eff }}$ [see, for example, Eq. (11)], while the strength is proportional to $\beta$. Keeping the environment parameters fixed, the longer the error cycle, the longer the range of the interactions, as qubit correlations are intermediated by the propagation of

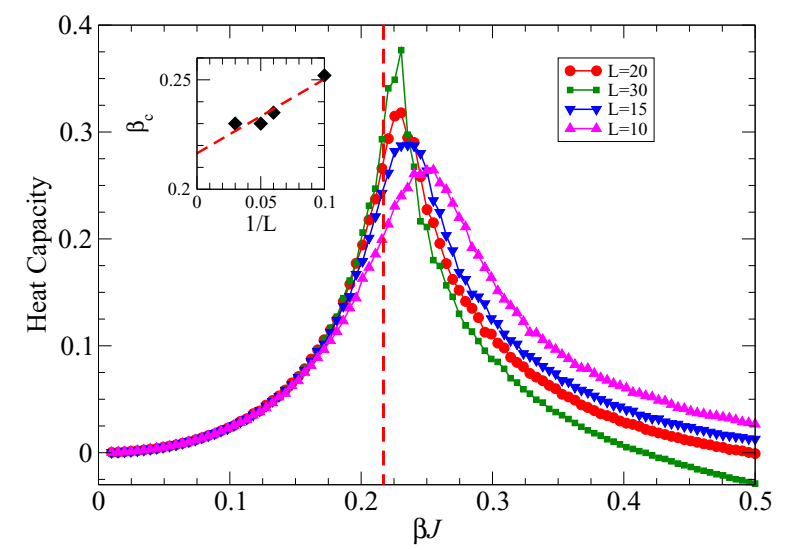

FIG. 5. (Color online) Finite-size scaling analysis of the heat capacity per qubit that yields $\beta_{c}=0.217$ for $L \rightarrow \infty$. The solid lines are guides to the eye.

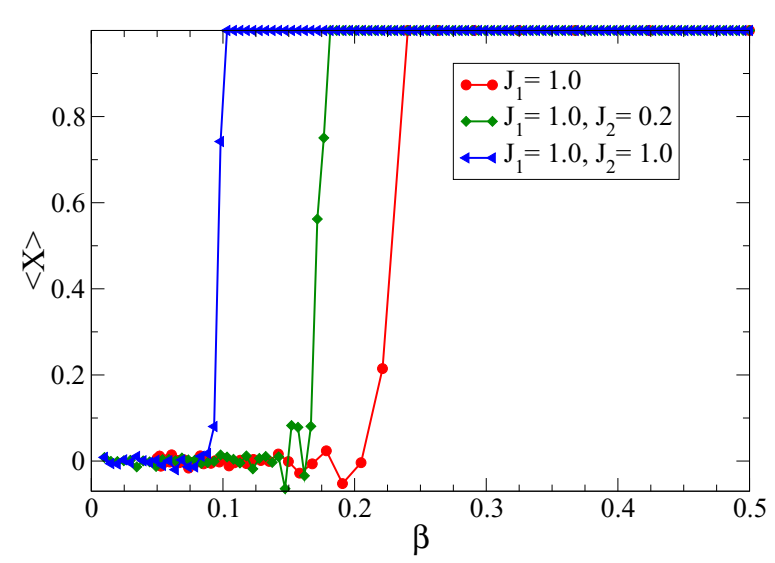

FIG. 6. (Color online) The ratio $\left|\frac{\mathcal{B}}{A}\right|$ for different interaction ranges as a function of $\beta$ on a lattice with $L=40$ and 80000 Monte Carlo steps for each data point. The data points correspond to $J_{1}=1$ (circles), as in Fig. $4 ; J_{1}=1, J_{2}=0.2$, and $J_{m}=0$ for $m>2$ (diamonds); and $J_{1}=1, J_{2}=1$, and $J_{m}=0$ for $m>2$ (triangles) in Eq. (38). The solid lines are guides to the eye.

environmental modes. To extend the analysis to qubit-qubit interactions beyond nearest neighbors, we write

$$
H_{\mathrm{eff}}=-\sum_{m} \sum_{m \text {-th n.n }(i j)} J_{m} \sigma_{i}^{x} \sigma_{j}^{x},
$$

where $J_{m}$ is the exchange coupling between $m$ th nearest neighbors (see Fig. 2). In Fig. 6 the behavior of $\left|\frac{\mathcal{B}}{\mathcal{A}}\right|$ as a function of $\beta$ is shown for some fixed values of $J_{m}$. By increasing $\Delta$ the interaction range in $H_{\text {eff }}$ varies and therefore one needs to take into account higher orders of $m$ in Eq. (38). By including higher orders of $m$, the threshold value in the coupling to the environment for which the code protection is lost also changes. We see that a longer QEC cycle brings a larger range of correlated errors into account and consequently decreases the threshold value $\beta_{c}$. This indicates that for increasing values of $\Delta$, a smaller coupling to the bath is sufficient to destroy the topological state of the surface code. We should emphasize that the effect is robust against increases of system size and the value of $\beta_{c}$ is also well defined in the thermodynamic limit in this case. In general, the dependence of $J_{m}$ on $\Delta$ is determined by the characteristics (correlation functions) of the environment; for bosonic environments this dependence was derived for some representative cases in Ref. [20]. We have numerically calculated the ratio $\left|\frac{\mathcal{B}}{\mathcal{A}}\right|$ for interaction ranges up to the fourth nearest neighbor. The results (not shown) confirm a trend of decreasing thresholds when the interaction range is increased.

\section{B. Results for one-error sector $\mathcal{P}_{1, f}$ and the two-error sector $\mathcal{P}_{2, f}$}

To investigate the intrinsic nature of the transition mentioned above we have also numerically evaluated $\left\langle S_{1,2}^{x}\right\rangle=\left|\frac{\mathcal{B}}{\mathcal{A}}\right|$ for charge sectors $\mathcal{P}_{1, f}$ (where a plaquette $B_{p_{0}}$ is measured to be -1 ) and $\mathcal{P}_{2, f}$ (where two plaquettes $B_{p_{1}}$ and $B_{p_{2}}$ are measured -1$)$. The locations of the errors $\left\{B_{p_{0}}\right\}$ and $\left\{B_{p_{1}}, B_{p_{2}}\right\}$ are arbitrarily chosen as shown in Fig. 7. 

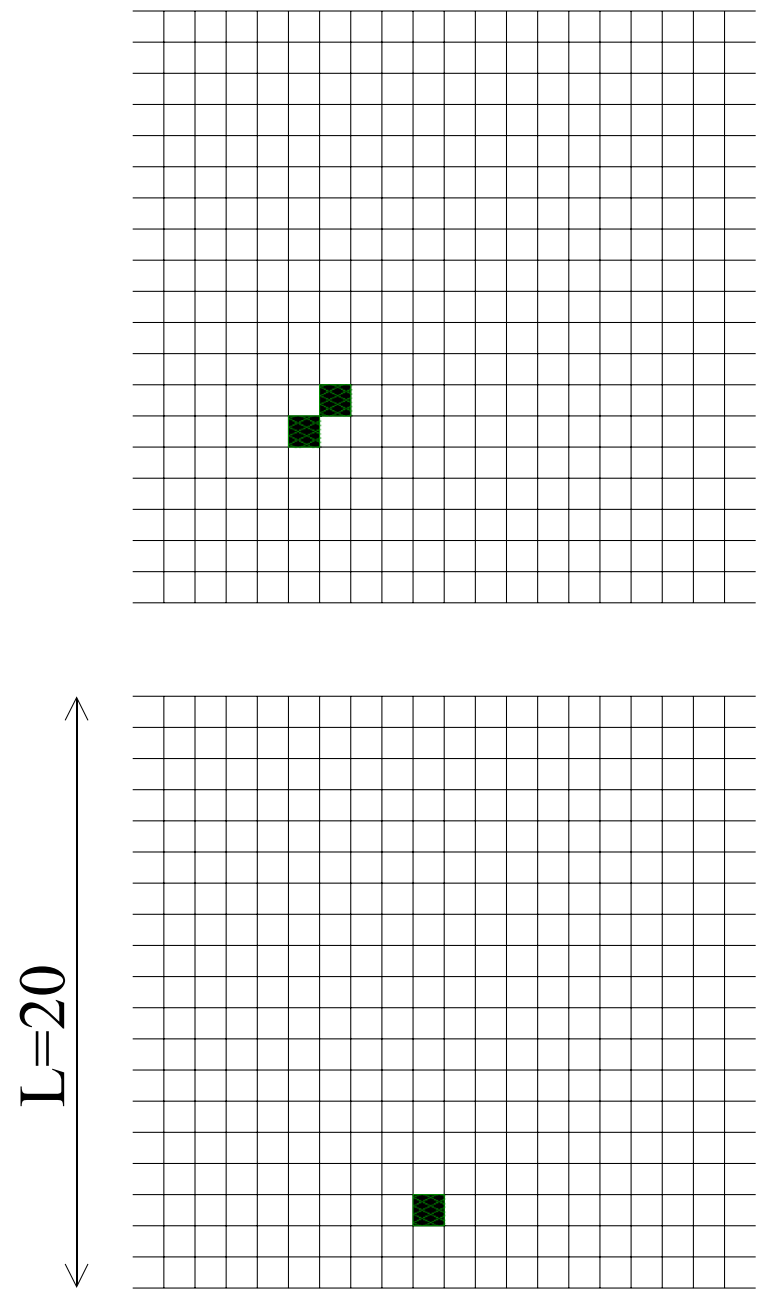

FIG. 7. (Color online) The location of the single error $\left\{B_{p_{0}}\right\}$ (bottom) and two errors $\left\{B_{p_{1}}, B_{p_{2}}\right\}$ (top) assumed in the numerical calculations.

In the presence of detected errors, the numerical calculations require a higher number of iterations. Figure 8 shows the gradual convergence of the results for the oneand two-error sectors to the results achieved for the no-error sector. In these calculations only the nearest-neighbor case $\left(J_{1}=1\right)$ has been considered. As can be seen in Fig. 8, for small values of $\beta$, complete convergence is not achieved when the number of iterations is just $O\left(10^{4}\right)$ per data point and a much larger data set is required. However, the data shows a clear tendency of convergence toward the same curve obtained for the no-error sector when the number of iterations is increased. The results for the case with a larger range of correlated errors $\left[J_{m} \neq 0\right.$ for $m>1$ in Eq. (38)] converge toward their counterpart of no-error syndrome as well (not shown). In fact, we observe a faster convergence when the range of correlations is larger. Results for other nonzero error configurations different than the configurations considered here were found to be consistence with the results shown in Fig. 8. However, a larger distance between errors requires a significantly higher number of computational iterations to achieve convergence.
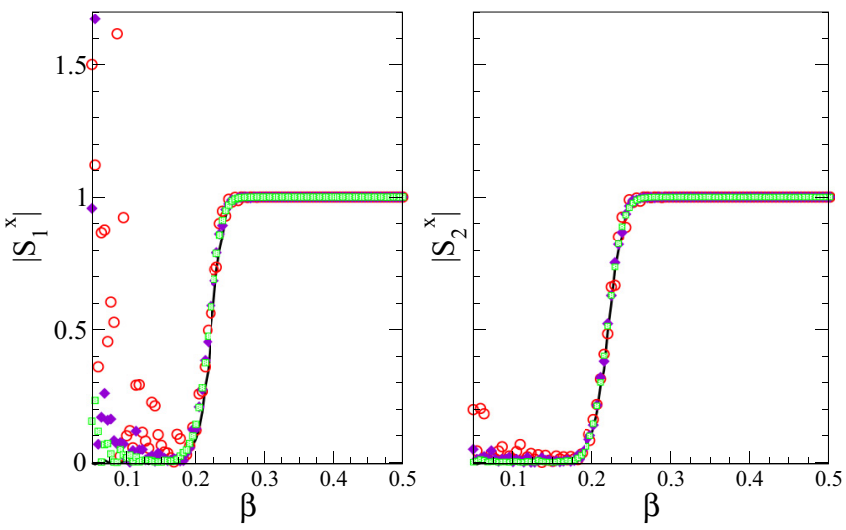

FIG. 8. (Color online) The ratio $\left|\frac{\mathcal{B}}{A}\right|$ for a lattice of $L=20$. The left box shows the convergence of $\left\langle S_{1}^{x}\right\rangle=\left|\frac{\mathcal{B}}{\mathcal{A}}\right|$ for one detected error located as shown in Fig. 7. The right box shows the convergence of $\left\langle S_{2}^{x}\right\rangle=\left|\frac{\mathcal{B}}{\mathcal{A}}\right|$ for two detected errors located as shown in Fig. 7. In both boxes the number of iterations used for each $\beta$ data point is 90000 (circle), 180000 (square), and 900000 (diamonds). The solid line is obtained from the corresponding no-error sector (Fig. 6).

\section{SUMMARY}

A non-Markovian environment in contact with the surface code induces an effective dynamics (action) on the code system. Environmental degrees of freedom can intermediate interactions between physical qubits making up the system. As a result, when errors occur during the code evolution, they will be correlated. We considered the effect of such correlated errors on the fidelity of the code state after one error correction cycle. We studied the code state resulting from an effective action derived for a gapless bosonic environment but expect the same model to describe phenomenologically other types of environments. The calculation of the expectation values that enter in the fidelity can be cast in the form of expectation values of a statistical mechanics spin model with two separate phases. The disordered and ordered phases of the statistical version correspond to the topological and nontopological states of the surface code system in contact with the environment. We evaluated an upper bound for the threshold of the coupling to the bath beyond which no quantum error correction is possible (i.e., fidelity is fully lost).

We showed numerically that the transition between the two phases can be evaluated by a Monte Carlo method. We used a definition for the stabilizers of the code based on the logical errors. The logical error in this definition plays a role equivalent to a world-line in the world-line-based quantum Monte Carlo. The separation of the two phases of the surface code lies behind the statistical physics of these world-lines, as presented in Eqs. (36) and (37). The alternative definition for stabilizers given in this paper can be extended to higher-dimensional topological codes where the stabilizers are defined on hypercubes and logical errors correspond to closed hypersurfaces [26]. In higher-dimensional codes the stabilizers can be defined in terms of the logical errors similar to Eqs. (33) and (34). Therefore, a similar approach should be applicable to those codes.

In the numerical evaluation we considered qubit-qubit interactions with different interaction ranges. We considered a 
QEC cycle with both zero and nonzero error syndromes. For the nearest-neighbor range the results perfectly agrees with the analytical calculations in Ref. [25]. Finite-size scaling shows the value for the threshold $\beta_{c}$ to be close to half of an Ising model with nearest-neighbor interaction. For a longer range of interactions the threshold $\beta_{c}$ decreases. The type of error syndrome does not affect the value $\beta_{c}$. However, higher numerical iterations were required to achieve convergence beyond nonerror syndromes.

Our results are based on the assumption that the interaction between the physical qubits and the environment has the form $V_{B C}=\lambda \sum_{r_{i}} f\left(r_{i}\right) \sigma_{i}^{x}$. Different functional forms for this interaction may result in a different effective evolution operator $U_{\text {eff }}$ than the one studied here and may set different threshold values for the fidelity. Another question that should be addressed is the behavior of the fidelity over multiple QEC cycles. Both issues are open to future investigations.

\section{ACKNOWLEDGMENTS}

We thank Robert Raussendorf, Igor Tupitsyn, and Eduardo Novais for insightful conversations. This work was supported in part by the office of Naval Research and the National Science Foundation under Grant No. CCF-1117241.
[1] A. Kitaev, Ann. Phys. (NY) 303, 2 (2003).

[2] E. Dennis, A. Kitaev, A. Landahl, and J. Preskill, J. Math. Phys. (NY) 43, 4452 (2002).

[3] M. H. Freedman, A. Kitaev, M. J. Larsen, and Z. Wang, Am. Math. Soc. 40, 31 (2003).

[4] C. Nayak, S. H. Simon, A. Stern, M. Freedmann, and S. Das Sarma, Rev. Mod. Phys. 80, 1083 (2008).

[5] S. B. Bravyi and A. Yu. Kitaev, arXiv:cond-mat/9811052.

[6] M. H. Freedman and D. A. Meyer, arXiv:cond-mat/9810055.

[7] J. Clarke and F. K. Wilhelm, Nature (London) 453, 1031 (2008); D. P. DiVincenzo, Phys. Scr. T 137, 014020 (2009).

[8] B. M. Terhal, F. Hassler, and D. P. DiVincenzo, Phys. Rev. Lett. 108, 260504 (2012).

[9] H. Paik et al., Phys. Rev. Lett. 107, 240501 (2011).

[10] J. M. Martinis, K. B. Cooper, R. McDermott, M. Steffen, M. Ansmann, K. D. Osborn, K. Cicak, S. Oh, D. P. Pappas, R. W. Simmonds, and C. C. Yu, Phys. Rev. Lett. 95, 210503 (2005).

[11] R. Barends et al., Nature (London) 508, 500 (2014).

[12] R. Raussendorf and J. Harrington, Phys. Rev. Lett. 98, 190504 (2007).

[13] A. G. Fowler, A. M. Stephens, and P. Groszkowski, Phys. Rev. A 80, 052312 (2009).

[14] A. Hutter and D. Loss, Phys. Rev. A 89, 042334 (2014).
[15] J. R. Wootton and D. Loss, Phys. Rev. Lett. 109, 160503 (2012).

[16] B. M. Terhal, arXiv:1302.3428.

[17] H. Bombin, R. S. Andrist, M. Ohzeki, H. G. Katzgraber, and M. A. Martin-Delgado, Phys. Rev. X 2, 021004 (2012).

[18] J. Preskill, Technical Report No. CALT 68-2881 (California Institute of Technology, Pasadena, 2012); Quant. Inf. Comput. 13, 181 (2013).

[19] E. Novais and E. R. Mucciolo, Phys. Rev. Lett. 110, 010502 (2013).

[20] P. Jouzdani, E. Novais, and E. R. Mucciolo, Phys. Rev. A 88, 012336 (2013).

[21] F. L. Pedrocchi, A. Hutter, J. R. Wootton, and D. Loss, Phys. Rev. A 88, 062313 (2013).

[22] A. Hamma, C. Castelnovo, and C. Chamon, Phys. Rev. B 79, 245122 (2009).

[23] M. Kardar, Statistical Physics of Fields (Cambridge University Press, Cambridge, 2007).

[24] F. F. Assaad and H. G. Evertz, in Computational Many-Particle Physics, edited by H. Fehske, R. Schneider, and A. Weisse, Vol. 739 (Springer, Berlin, Heidelberg, 2008).

[25] P. Jouzdani, E. Novais, I. S. Tupitsyn, R. Raussendorf, and E. R. Mucciolo, arXiv:1401.6540.

[26] C. Castelnovo and C. Chamon, Phys. Rev. B 78, 155120 (2008); S. Bravyi and B. Terhal, New J. Phys. 11, 043029 (2009). 